\title{
Systematic review and meta-analysis of randomized clinical trials comparing efficacy and safety outcomes of insulin glargine with NPH insulin, premixed insulin preparations or with insulin detemir in type 2 diabetes mellitus
}

\author{
Przemyslaw Rys $\cdot$ Piotr Wojciechowski $\cdot$ \\ Agnieszka Rogoz-Sitek • Grzegorz Niesyczyński • \\ Joanna Lis · Albert Syta • Maciej T. Malecki \\ Received: 22 March 2014/ Accepted: 9 December 2014/Published online: 14 January 2015 \\ (C) The Author(s) 2015. This article is published with open access at Springerlink.com
}

\begin{abstract}
Aims A variety of basal insulin preparations are used to treat patients with type 2 diabetes mellitus (T2DM). We aimed to summarize scientific evidence on relative efficacy and safety of insulin glargine (IGlar) and other insulins in T2DM.

Methods A systematic review was carried out in major medical databases up to December 2012. Relevant studies compared efficacy and safety of IGlar, added to oral drugs (OAD) or/and in combination with bolus insulin, with protamine insulin (NPH) or premixed insulin (MIX) in the same regimen, as well as with insulin detemir (IDet), in T2DM. Target HbA1c level without hypoglycemic events was considered the primary endpoint.

Results Twenty eight RCTs involving 12,669 T2DM patients followed for 12-52 weeks were included in quantitative analysis. IGlar + OAD use was associated with
\end{abstract}

Managed by Massimo Federici.

Electronic supplementary material The online version of this article (doi:10.1007/s00592-014-0698-4) contains supplementary material, which is available to authorized users.

P. Rys · P. Wojciechowski · A. Rogoz-Sitek · G. Niesyczyński HTA Consulting, Kraków, Poland

J. Lis - A. Syta

Sanofi-Aventis, Warsaw, Poland

M. T. Malecki $(\square)$

Department of Metabolic Diseases, Jagiellonian University

Medical College, 15 Kopernika Street, 31-501 Kraków, Poland

e-mail: malecki_malecki@yahoo.com;

maciej.malecki@uj.edu.pl

M. T. Malecki

University Hospital, Kraków, Poland higher probability of reaching target $\mathrm{HbA1c}$ level without hypoglycemia as compared to NPH $+\mathrm{OAD}(\mathrm{RR}=1.32$ $[1.09,1.59])$ or MIX without $\mathrm{OAD}(\mathrm{RR}=1.61[1.22,2.13])$ and similar effect as IDet $+\mathrm{OAD}(\mathrm{RR}=1.07[0.87,1.33])$ and MIX + OAD $(\mathrm{RR}=1.09[0.86,1.38])$. IGlar + OAD demonstrated significantly lower risk of symptomatic hypoglycemia as compared to $\mathrm{NPH}+\mathrm{OAD}(\mathrm{RR}=0.89$ $[0.83,0.96])$, MIX + OAD $(\mathrm{RR}=0.75[0.68,0.83])$ and MIX without $\mathrm{OAD}(\mathrm{RR}=0.75[0.68,0.83])$, but not with IDet + OAD $(\mathrm{RR}=0.99[0.90,1.08])$. In basal-bolus regimens, IGlar demonstrated similar proportion of T2DM patients achieving target $\mathrm{HbAlc}$ as compared to NPH $(\mathrm{RR}=1.14[0.91,1.44])$ but higher than $\mathrm{MIX}(\mathrm{RR}=1.26$ $[1.12,1.42)$ or IDet $(\mathrm{RR}=1.38[1.11,1.72])$. The risk of severe hypoglycemia was lower in IGlar than in NPH $(\mathrm{RR}=0.77[0.63,0.94])$, with no differences in comparison with MIX $(\mathrm{RR}=0.74[0.46,1.20])$ and IDet $(\mathrm{RR}=1.10$ $[0.54,2.25])$. IGlar + OAD has comparable safety profile to $\mathrm{NPH}$, with less frequent adverse events leading to treatment discontinuation than MIX + OAD $(\mathrm{RR}=0.41[0.22,0.76])$ and IDet + OAD $(\mathrm{RR}=0.40[0.24,0.69])$. Also severe adverse reactions were less common for IGlar + OAD when compared to MIX + OAD ( $\mathrm{RR}=0.71[0.52 ; 0.98])$. Conclusion For the majority of examined efficacy and safety outcomes, IGlar use in T2DM patients was superior or non-inferior to the alternative insulin treatment options.

Keywords Type 2 diabetes mellitus - Insulin therapy · Insulin glargine $\cdot$ Long-acting insulin analog

\section{Introduction}

Type 2 diabetes mellitus (T2DM) is a progressive disease, which requires insulin treatment when other management 
is no longer effective. Appropriate insulin therapy should be chosen individually to patient's needs in order to achieve treatment goals and maintain its safety $[1,2]$. In clinical practice, the flexibility of insulin titration is limited by the associated risk of hypoglycemic events, particularly when intensive insulin treatment is required [3, 4]. A growing body of evidence revealed that hypoglycemia is a predictor of poor outcome in people with T2DM, particularly it increases the risk of premature death [5-7]. Therefore, most of the clinical practice recommendations highlight that the optimal glycemic control in T2DM patients should be achieved with minimized risk of hypoglycemia $[2,8]$.

In general, it is recommended that at the initiation of insulin treatment in T2DM, once daily basal insulin is added to oral antidiabetic drugs (OADs) [1, 9-11]. Neutral protamine Hagedorn (NPH) has been frequently chosen as the first-line insulin; however, its use is associated with the risk of both hyper- and hypoglycemic events [12, 13]. Long-acting insulin analogs (LAAs) have been developed by modification of insulin chain in order to improve pharmacokinetic properties and decrease the risk of hypoglycemia. The first developed and most commonly prescribed LAA product is glargine (IGlar) [14]. Following the injection, IGlar forms a depot in the subcutaneous tissue, from which it is slowly absorbed. This provides a relatively uniform concentration over approximately $24 \mathrm{~h}$ after administration, which allows mimicking basal endogenous insulin secretion [12].

Complex pathophysiology of T2DM, its progressive nature, heterogeneous clinical picture and concomitant diseases require a variety of therapeutic options, including plural insulin regimens in order to maintain appropriate glycemic control and treatment safety. IGlar is frequently used as once daily regimen in addition to OAD. Interestingly, it has been demonstrated that early basal insulin initiation with IGlar improves FPG control and beta-cell function when compared to prolonged continuation of solely oral therapy [15]. When necessary, prandial insulin preparations can be used to intensify treatment. Therefore, IGlar is suitable for a spectrum of treatment intensities and can be used in T2DM patients at different age and various stages requiring insulin.

So far, several attempts have been undertaken to provide reliable summary of data comparing IGlar with other therapeutic options in T2DM patients [16-34]. However, available systematic reviews have assessed only selected insulin preparations and do not provide a broad clinical picture or comprehensive answer, whether IGlar use is associated with additional clinical benefits to a wide spectrum of T2DM patients. Here, for the first time, we performed a systematic review combining all data from randomized clinical trials (RCTs) in T2DM to compare efficacy and safety outcomes of IGlar with several other insulin regimens in order to make synthetic and reliable conclusions.

\section{Methods}

\section{Search strategy}

Following the preferred reporting items for systematic reviews and meta-analyses (PRISMA) guidelines, clinical evidence was identified through a systematic search of major databases of medical information, including Medline (via PubMed), EMBASE and the Cochrane Central Register of Controlled Trials [35]. The search strategy was constructed by combining search terms with appropriate Boolean operators in order to describe records including key words referring to both diabetes mellitus and IGlar. Databases were searched until December, 2012. Clinical trials registers (clinicaltrials.gov, ISRCTN.org) and abstracts presented at international meetings organized by the associations active in the field of diabetes (ADA, EASD) were screened for the most up-to-date clinical studies. Furthermore, references of identified articles, the websites of US Food and Drug Administration (FDA), the European Medicines Agency (EMA), Medicines and Healthcare Products Regulatory Agency (MHRA) were screened in order to retrieve potentially relevant data.

\section{Inclusion and exclusion criteria}

Studies enrolling adults with T2DM were included. Fulltext publications were considered eligible when reported RCTs directly comparing IGlar, added to OAD or/and in combination with bolus insulin, with human insulin (NPH) or insulin detemir (IDet) in the same regimens, as well as with premixed insulin (MIX). Relevant trials had to have parallel design with at least 12 weeks of follow-up; however, results of interest from the first period of cross-over studies were also accepted.

Studies enrolling a mixed population of patients with both T1DM and T2DM were excluded unless they presented separate data for the subset of individuals with T2DM. Trials recruiting only patients with non-caucasian ethnicity were not considered as race may potentially influence the effects of insulin therapy [36].

Study selection and credibility assessment

Two analysts worked independently to select relevant studies at each stage of selection process, starting from screening of abstracts and titles and ending on thorough analysis of full texts together with credibility assessment. 
Discrepancies between analysts were solved by consensus. Credibility of included trials was assessed according to the scale proposed by Jadad et al., which granted from 0 to 5 points according to the presence and accuracy of methods for randomization and double blinding, and accuracy of information regarding patients lost to follow-up. Higher number of granted points reflected higher credibility of a clinical trial [37].

\section{Outcome of interest}

The primary endpoint was glycemic control defined as a composite of target HbA1c level of $\leq 7 \%(53 \mathrm{mmol} / \mathrm{mol})$ without hypoglycemia. Due to differences in definitions of outcomes assessed in respective studies, the composite endpoint in this analysis encompassed either overall, severe or nocturnal hypoglycemic events; nevertheless, only homogenous results were allowed for statistical accumulation.

Key secondary endpoints in efficacy analysis included glycemic control, expressed either as the absolute reduction in mean value of glycemic parameters or percentage of patients achieving target values of $\mathrm{HbA1c}$ of $\leq 7 \%$ $(53 \mathrm{mmol} / \mathrm{mol})$. Treatment satisfaction and quality of life were also assessed. The risk of hypoglycemic episodes as well as mean weight gain during treatment was analyzed separately. Furthermore, safety analysis was conducted, which assessed the number of patients with at least one adverse event, serious adverse event and number of subjects who prematurely withdrew from the study due to safety reason.

\section{Statistical analysis}

Dichotomous effect measures were presented as relative risk (RR), while continuous endpoints were assessed with weighted mean difference (WMD). All estimates were presented together with $95 \%$ confidence intervals. Between-study heterogeneity was examined using the Cochran Q test and the $I^{2}$ statistics and was considered significant when either $p<0.1$ or $I^{2} \geq 50 \%$. When homogeneity was confirmed, dichotomous and continuous variables were accumulated using fixed effects model with Mantel-Haenszel or inverse-variance methods, respectively. In case of statistically significant heterogeneity, DerSimonian and Laird random effect model was performed both for continuous and for dichotomous outcomes [38]. Significance of the overall effect was tested with Z-test assuming $p<0.05$ as the level of significance. The results were processed using Sophie version 1.5.0 (meta-analysis software by HTA Consulting-verified and producing consistent results with STATA version 10.0).

\section{Results}

A total number of 3,186 records without duplicates were identified in the systematic search of medical databases. After the screening of titles and abstracts, 430 studies were considered potentially relevant and were subjected for further assessment based on full-text publications. A total number of 363 studies were subsequently excluded from the analysis due to reasons presented in PRISMA diagram (Fig. 1). Finally, 29 RCTs were included in qualitative and 28 in quantitative analysis. Overall, studies included in quantitative analysis enrolled 12,669 T2DM patients, who were followed for 12-52 weeks (Tables 1, 2).

Nine studies compared IGlar versus NPH [39-47], while in two others, patients from the comparatory group received neutral protaminated insulin lispro (NPL) $[48,49]$. However, all these studies were analyzed together as NPL demonstrates similar pharmacokinetic and pharmacodynamic properties to NPH [50]. In nine studies, protamine insulin was administered once daily, while in the remaining two trials, patients were allowed to receive protaminated insulin according to either $q d$ or bid schemes [47, 49]. Nine studies assessed basal + OAD regimen; in four of which patients received one oral drug either metformin [40,43] or a sulphonylurea derivative [41, 42], while in the remaining five RCTs participants could be treated with more than one OAD $[39,44,45,48,49]$. In two studies assessing basal + bolus regimen, either human or lispro insulin was used as prandial insulin and OAD therapy was allowed but not obligatory [46, 47]. Additionally, one long-term RCT comparing IGlar + OAD with NPH + OAD was identified. However, this study was not included in quantitative analysis due to heterogeneous treatment and much longer follow-up (260 weeks) when compared to the remaining RCTs [51].

Thirteen studies assessed IGlar in comparison with MIX, of which three compared IGlar + OAD with MIX monotherapy [52-54], while in eight of them patients received insulins in combination with OAD in both groups [55-59]. Remaining 5 RCTs assessed IGlar + bolus \pm OAD regimen in comparison with MIX \pm OAD [60-64]. Premixed insulin analogs were used as comparators in most RCTs, except for participants of trials comparing IGlar + OAD with mix monotherapy [52-54] and $59 \%$ of subjects from another study assessing basal + bolus regimen in comparison with MIX [62], who were treated with human premixed insulins.

Finally, four studies assessed IGlar in comparison with IDet. Both LAA were administered in basal + OAD regiment in two RCTs $[65,66]$ and according to basal + bolus scheme in the remaining two $[67,68]$.

In most studies, the follow-up was not longer than 6 months, while three studies reported the outcomes after around 1 year of treatment (Table 2) [45, 60, 62]. The credibility of included RCTs, assessed according to the 
Fig. 1 PRISMA diagram for study selection process

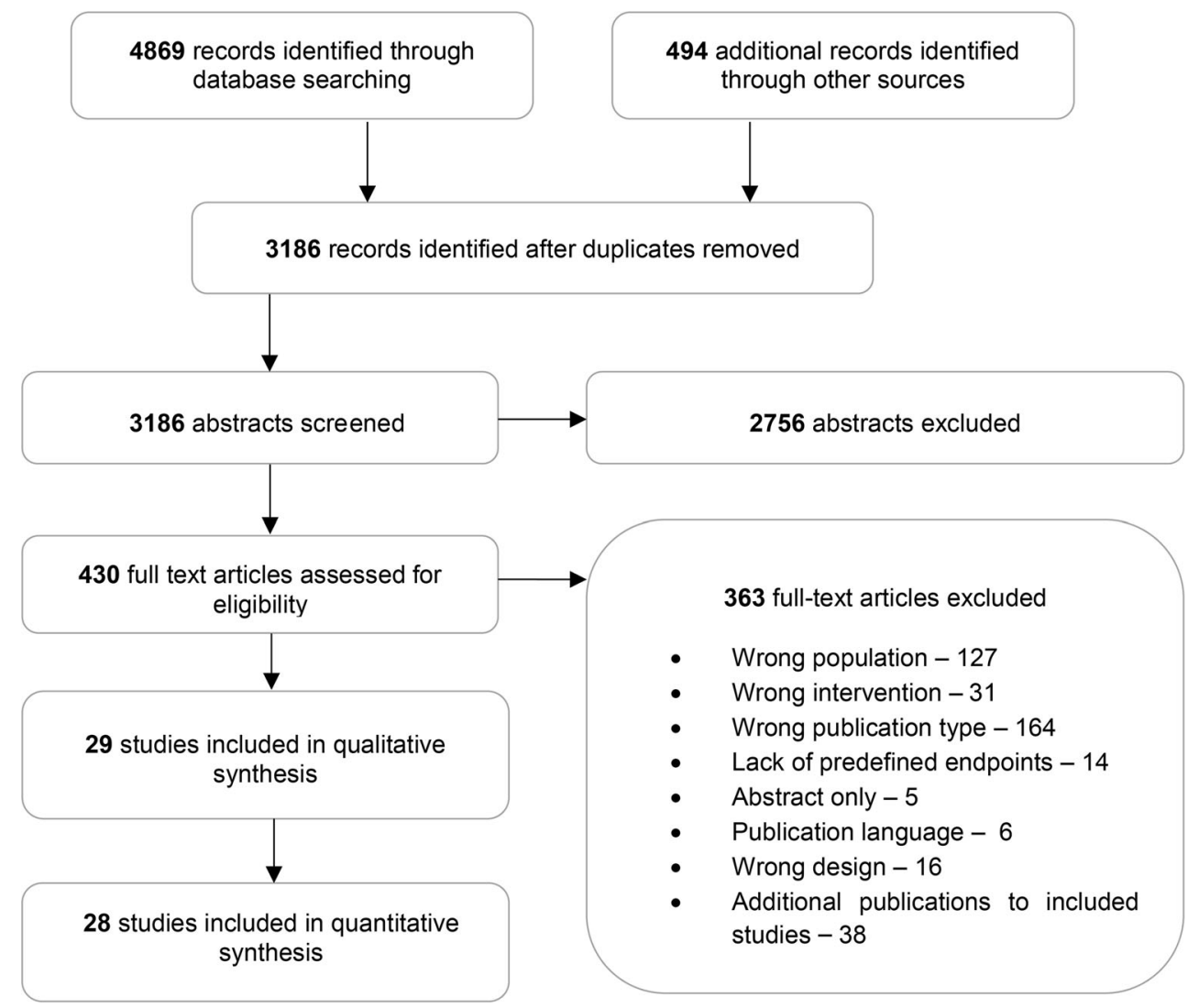

Jadad scale, oscillated between 1 and 3 points on the 5 -point scale and was mainly downgraded due to the lack of double blinding (Table 1).

\section{IGlar versus NPH}

\section{Glycemic control}

The meta-analysis of 2 RCTs assessing basal + OAD regimen demonstrated a favorable effect of IGlar over NPH with respect to target $\mathrm{HbA} 1 \mathrm{c}$ without nocturnal hypoglycemia $(\mathrm{RR}=1.32[1.09,1.59])$, while the mean reduction in HbA1c level was comparable in both arms (9 RCTs; $\mathrm{WMD}=-0.03 \%[-0.10,0.04](-0.3 \mathrm{mmol} / \mathrm{mol}[-1.1$; $0.4])$ ). No difference between IGlar and NPH, both in combination with prandial insulin, was observed with respect to the mean reduction of HbAlc (2 RCTs; $\mathrm{WMD}=0.02 \% \quad[-0.30,0.35] \quad(0.2 \mathrm{mmol} / \mathrm{mol} \quad[-3.3$; 3.8])) as well as the number of T2DM patients achieving target HbAlc (1 RCT; RB = 1.14 [0.91; 1.44]) [46].

\section{Hypoglycemia}

Meta-analysis of five studies assessing IGlar in comparison with NPH, both added to OAD, revealed a borderline difference toward lower risk of overall hypoglycemia in patients treated with $\operatorname{IGlar}(\mathrm{RR}=0.92$ [0.84, 1.001]). Moreover, IGlar + OAD significantly reduced number of patients experiencing symptomatic (6 RCTs; $\mathrm{RR}=0.89[0.83,0.96])$ and nocturnal events (6 RCTs; $\mathrm{RR}=0.63[0.51 ; 0.77])$. The risk of severe hypoglycemia was comparable between interventions (5 RCTs; RR =0.76 [0.47, 1.23]) [41, 42, 44, 45, 49].

Meta-analysis of the 2 RCTs assessing basal + bolus scheme demonstrated less frequent nocturnal hypoglycemic events in patients treated with IGlar as compared to protamine insulin $(\mathrm{RR}=0.77$ [0.63, 0.94]). Additionally, a tendency toward lower risk of severe hypoglycemic events was shown in patients treated with IGlar $(\mathrm{RR}=0.22[0.05$, 1.02]) [46, 47].

\section{Weight gain}

IGlar and NPH did not differ significantly with respect to weight gain when administered within basal + OAD (6 RCTs; WMD $=0.36 \mathrm{~kg}[-0.12,0.84]$ ) or basal + bolus \pm OAD regimens $(2$ RCTs; WMD $=-0.45 \mathrm{~kg}$ $[-1.52,0.61])$. 
Table 1 Study quality and risk of bias assessment

\begin{tabular}{|c|c|c|c|c|c|c|c|}
\hline Study & Sponsor & $\begin{array}{l}\text { Method of } \\
\text { randomization }\end{array}$ & $\begin{array}{l}\text { Double } \\
\text { blinding }\end{array}$ & $\begin{array}{l}\text { Description } \\
\text { of lost to } \\
\text { follow-up }\end{array}$ & $\begin{array}{l}\text { Allocation } \\
\text { concealment }\end{array}$ & $\begin{array}{l}\text { Type of } \\
\text { analysis }\end{array}$ & $\begin{array}{l}\text { Total } \\
\text { Jadad } \\
\text { score }\end{array}$ \\
\hline \multicolumn{8}{|c|}{ IGlar + OAD versus $\mathrm{NPH}+\mathrm{OAD}$} \\
\hline Hsia [39] & NIH & No description & Open labeled & Sufficient & Unclear & ITT; PP & $2 / 5$ \\
\hline Forst $[40]$ & Sanofi-Aventis & No description & Open labeled & Sufficient & Unclear & PP & $2 / 5$ \\
\hline Esposito [48] & Second University of Naples & Adequate & Open labeled & Sufficient & Adequate & PP & $3 / 5$ \\
\hline Eliaschewitz [41] & Sanofi-Aventis & No description & Open labeled & Sufficient & Unclear & mITT & $2 / 5$ \\
\hline Fritsche [42] & Sanofi-Aventis & Adequate & Open labeled & Sufficient & Unclear & mITT & $3 / 5$ \\
\hline $\begin{array}{l}\text { Yki-Järvinen } \\
\text { [43] }\end{array}$ & Sanofi-Aventis & Adequate & Open labeled & Sufficient & Unclear & mITT & $3 / 5$ \\
\hline Riddle [44] & Sanofi-Aventis & Adequate & Open labeled & Sufficient & Adequate & mITT & $3 / 5$ \\
\hline $\begin{array}{l}\text { Massi-Bendetti } \\
\text { [45] }\end{array}$ & $\begin{array}{l}\text { Grant from Hoechst Marion Russel } \\
\text { Deutschland Clinical Development }\end{array}$ & Adequate & Open labeled & Sufficient & Adequate & mITT & $3 / 5$ \\
\hline Strojek [49] & Eli Lilly & No description & Open labeled & Sufficient & Unclear & mITT & $2 / 5$ \\
\hline \multicolumn{8}{|c|}{ IGlar + bolus \pm OAD versus $\mathrm{NPH}+$ bolus \pm OAD } \\
\hline Rosenstock [47] & Sanofi-Aventis & No description & Open labeled & Sufficient & Unclear & ITT & $2 / 5$ \\
\hline Koivisto [46] & Eli Lilly & No description & Open labeled & Sufficient & Unclear & $\begin{array}{l}\mathrm{mITT} \text { and } \\
\mathrm{PP} / \mathrm{mITT}\end{array}$ & $2 / 5$ \\
\hline \multicolumn{8}{|c|}{ IGlar $+\mathrm{OAD} \pm$ bolus versus $\mathrm{NPH}+\mathrm{OAD} \pm$ bolus } \\
\hline Rosenstock [51] & Sanofi-Aventis & Adequate & Open labeled & Sufficient & Adequate & mITT; PP & $3 / 5$ \\
\hline \multicolumn{8}{|c|}{ IGlar + bolus \pm OAD versus IDet + bolus \pm OAD } \\
\hline Hollander [67] & Novo Nordisk & Adequate & Open labeled & Sufficient & Adequate & mITT & $3 / 5$ \\
\hline Raskin [68] & Novo Nordisk & No description & Open labeled & Insufficient & Unclear & mITT & $1 / 5$ \\
\hline \multicolumn{8}{|c|}{ IGlar + OAD versus IDet + OAD } \\
\hline Rosenstock [65] & Novo Nordisk & Adequate & Open labeled & Sufficient & Adequate & $\begin{array}{l}\text { ITT or } \\
\text { mITT/ITT }\end{array}$ & $3 / 5$ \\
\hline Swinnen [66] & Sanofi-Aventis & Adequate & Open labeled & Sufficient & Adequate & mITT, PP & $3 / 5$ \\
\hline \multicolumn{8}{|c|}{ IGlar + OAD versus MIX } \\
\hline Al-Shaikh [52] & $\mathrm{n} / \mathrm{a}$ & No description & Open labeled & Sufficient & Unclear & ITT & $2 / 5$ \\
\hline Janka [53] & Sanofi-Aventis & Adequate & Open labeled & Sufficient & Adequate & mITT & $3 / 5$ \\
\hline Schiel [54] & Sanofi-Aventis & Adequate & Open labeled & Sufficient & Adequate & mITT & $3 / 5$ \\
\hline \multicolumn{8}{|c|}{ IGlar + OAD versus MIX +OAD } \\
\hline $\begin{array}{l}\text { DURABLE } 1 \\
\text { [55] }\end{array}$ & Eli Lilly & Adequate & Open labeled & Sufficient & Adequate & $\begin{array}{l}\mathrm{mITT} / \\
\mathrm{mITT}\end{array}$ & $3 / 5$ \\
\hline Kann [56] & Novo Nordisk & $\begin{array}{l}\text { Properly } \\
\text { described }\end{array}$ & Open labeled & Insufficient & Unclear & mITT & $1 / 5$ \\
\hline Raskin [57] & Novo Nordisk & $\begin{array}{l}\text { Properly } \\
\text { described }\end{array}$ & Open labeled & Sufficient & Unclear & mITT & $2 / 5$ \\
\hline Robbins [58] & $\mathrm{n} / \mathrm{a}$ & Adequate & Open labeled & Sufficient & Adequate & mITT & $3 / 5$ \\
\hline Strojek [59] & Novo Nordisk & Adequate & Open labeled & Sufficient & Adequate & mITTi PP & $3 / 5$ \\
\hline \multicolumn{8}{|c|}{ IGlar + bolus \pm OAD versus $\mathrm{MIX} \pm \mathrm{OAD}$} \\
\hline Bowering [60] & Eli Lilly & No description & Open labeled & Sufficient & Unclear & $\begin{array}{c}\text { PP and } \\
\text { mITT/ } \\
\text { mITT }\end{array}$ & $2 / 5$ \\
\hline $\begin{array}{l}\text { DURABLE } 2 \\
\text { [61] }\end{array}$ & Eli Lilly & Adequate & Open labeled & Sufficient & Adequate & $\begin{array}{l}\text { mITT/ } \\
\text { mITT }\end{array}$ & $3 / 5$ \\
\hline GINGER [62] & Sanofi-Aventis & Adequate & Open labeled & Sufficient & Adequate & $\begin{array}{l}\mathrm{mITT} / \\
\mathrm{mITT}\end{array}$ & $3 / 5$ \\
\hline Jain [63] & Eli Lilly & Adequate & Open labeled & Sufficient & Adequate & ITT & $3 / 5$ \\
\hline Rosenstock [64] & Eli Lilly & Adequate & Open labeled & Sufficient & Adequate & PP/ITT & $3 / 5$ \\
\hline
\end{tabular}




\section{Treatment satisfaction and quality of life}

One RCT reported superior treatment satisfaction of IGlar over NPH, both added to OAD (WMD =0.60 [0.07; 1.13]) [41].

\section{Safety}

No difference between interventions was demonstrated with regard to the risk of adverse events related to study drug and the risk of study discontinuations due to adverse events. The incidence of serious adverse events (SAE) was generally low and did not reveal any difference between IGlar and NPH in either treatment regimens. Similarly, both basal insulins were associated with similar risk of overall adverse events when administered according to basal + OAD $(\mathrm{RR}=1.00[0.93,1.09])$ or basal + bolus \pm OAD $(1 \mathrm{RCT} ; \mathrm{RR}=1.13[0.88,1.44])$ regimens, respectively. Only single cases of mortality were reported in two RCTs comparing IGlar + OAD with NPH + OAD $[42,45]$, and in one RCT assessing both basal insulins in basal + OAD scheme with no differences between treatment arms [46].

IGlar versus premixed insulins (MIX)

\section{Glycemic control}

Single RCT reported that significantly more patients treated with IGlar + OAD achieved target HbA1c without nocturnal hypoglycemia when compared to MIX monotherapy $(\mathrm{RR}=1.61[1.22,2.13])$ [53]. Additionally, IGlar combined with OADs exerted a greater reduction in mean level of HbA1c (3 RCTs; WMD $=-0.36 \%$ [-0.54, $-0.18](-3.9 \mathrm{mmol} / \mathrm{mol}[-5.9 ;-2.0]))$ and was associated with a higher chance of reaching target HbAlc (2 RCTs; RR = 1.49 [1.03, 2.16]).

A single study demonstrated that both insulin preparations added to OAD have comparable efficacy with respect to primary endpoint defined as achievement of target HbAlc $<7 \% \quad(53 \mathrm{mmol} / \mathrm{mol})$ without either overall $(\mathrm{RR}=0.97[0.67,1.40])$ or nocturnal hypoglycemic events $(\mathrm{RR}=1.09[0.86,1.38])$ [59]. However, MIX + OAD provided larger reduction of HbA1c (5 RCTs; $\mathrm{WMD}=0.26 \%[0.12,0.40](2.8 \mathrm{mmol} / \mathrm{mol}[1.3,4.4]))$ and allowed to achieve target HbA1c in a higher number of patients (5 RCTs; RR $=0.79$ [0.66, 0.94]).

Meta-analysis of five studies demonstrated that IGlar added to prandial insulin compared with $\mathrm{MIX} \pm \mathrm{OAD}$ showed a trend toward lower mean HbA1c (WMD $-0.19 \%[-0.43,0.06](-2.1 \mathrm{mmol} / \mathrm{mol}[-4.7,0.7]))$ and was associated with a higher percentage of patients who reached target $\mathrm{HbA1c}(\mathrm{RR}=1.26[1.12,1.42])$.
Hypoglycemia

A meta-analysis of two studies comparing IGlar + OAD versus MIX monotherapy demonstrated no difference between groups with respect to the risk of overall hypoglycemia $(\mathrm{RR}=0.90$ [0.78; 1.04]) [53, 54]. However, Janka et al. [53] demonstrated significantly lower number of symptomatic (2.62 vs. 5.73 events/patient-year; $p<0.001)$ as well as nocturnal (0.051 vs. 1.04 events/ patient-year; $p<0.05$ ) hypoglycemic events in IGlar group. Severe hypoglycemia was uncommon in both arms $[53,54]$.

IGlar as compared to MIX, both administered together with OAD, demonstrated lower risk of overall (3 RCTs; $\mathrm{RR}=0.88[0.82,0.95])$ and symptomatic hypoglycemia (3 $\mathrm{RCTs} ; \mathrm{RR}=0.75$ [0.68, 0.83]), while no differences were found with respect to the risk of nocturnal (2 RCTs; $\mathrm{RR}=1.01[0.90,1.14])$ and severe events (5 RCTs; $\mathrm{RR}=0.86[0.30,4.43])[55,57-59]$.

IGlar added to prandial insulin when compared to MIX \pm OAD therapy demonstrated similar impact with respect to all assessed hypoglycemic endpoints including overall (2 RCTs; $\mathrm{RR}=1.01 \quad[0.93 ; 1.10])[62,63]$, symptomatic (2 RCTs; RR $=1.02[0.95 ; 1.10])[62,64]$, severe $\quad(5$ RCTs; $\mathrm{RR}=0.74 \quad[0.46,1.20])$ [60-64] and nocturnal events (3 RCTs; RR $=0.98[0.87 ; 1.10]$ ) [62-64].

\section{Weight gain}

Meta-analysis of three RCTs comparing IGlar added to OAD with MIX monotherapy demonstrated comparable weight gain in both groups (WMD $=-2.02 \mathrm{~kg}[-5.11$; 1.07]), although this result has limited credibility due to a significant between-study heterogeneity $(p=0.03)$ [5254].

Pooled estimate of three studies showed lower mean body weight gain in patients receiving IGlar + OAD than in those who were on MIX + OAD therapy (WMD = $-1.27 \mathrm{~kg}[-1.56,-0.97])[55,57,58]$. On the other hand, IGlar combined with prandial insulin provided comparable effect on weight gain as MIX \pm OAD (5 RCTs; $\mathrm{WMD}=0.37 \mathrm{~kg}[-0.20 ; 0,94])$.

\section{Treatment satisfaction and quality of life}

Irrespectively of assessed treatment scheme, no evidence was found for the difference in overall treatment satisfaction or quality of life between IGlar and MIX [54, 55, 60]. However, in one study IGlar + OAD provided withingroup improvement in hypoglycemic, cardiovascular and psychological/cognitive subdomains of DSC-R, while 


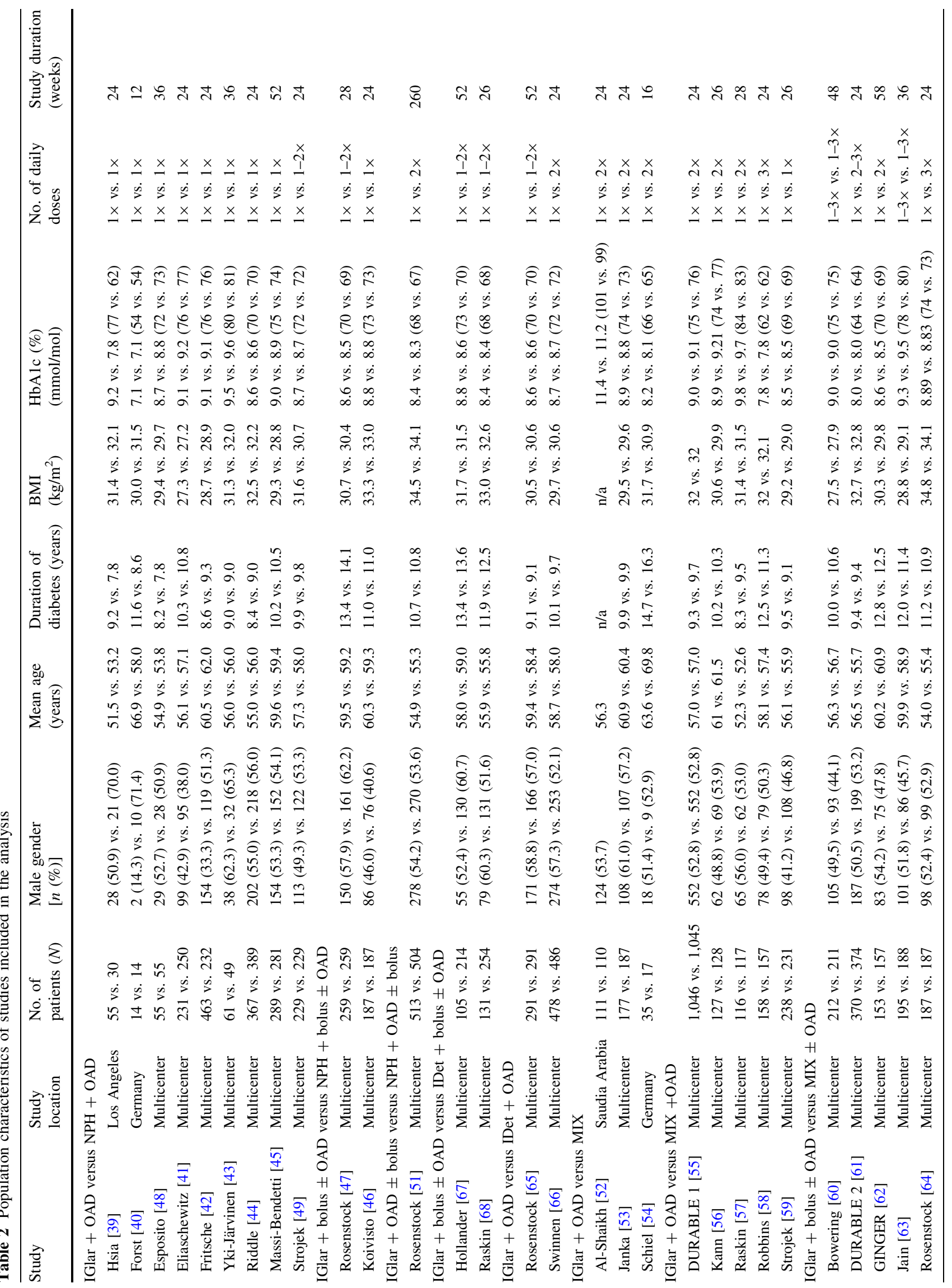


patients treated with MIX + OAD did not report significant difference from baseline [55].

\section{Safety}

The proportion of premature withdrawals due to adverse events was lower in IGlar + OAD group when compared to MIX + OAD (5 RCTs; RR = $0.41[0.22,0.76])$, but not to MIX monotherapy (2 RCTs; $\mathrm{RR}=0.52[0.13,1.99]$ ). Comparable number of withdrawals due to adverse events was observed for the comparison between IGlar + bolus \pm $\mathrm{OAD}$ vs. MIX $\pm \mathrm{OAD}$ (4 RCTs; RR $=1.44$ [0.63, 3.28]). In comparison with MIX + OAD, IGlar decreased the number of severe adverse events when used with OAD (3 $\mathrm{RCTs}$; $\mathrm{RR}=0.71[0.52,0.98])$, but not as adjunctive to prandial insulin (5 RCTs; RR $=1.05$ [0.78, 1.42]). Single cases of mortality were reported in 2 RCTs comparing IGlar with MIX, both added to OAD, and in 4 RCTs comparing IGlar + bolus \pm OAD versus $\mathrm{MIX} \pm \mathrm{OAD}$ with no difference between treatment arms. No evidence for the difference between IGlar and MIX with respect to both overall adverse events and treatment associated adverse events was found regardless of treatment schemes that were directly compared.

\section{IGlar versus IDet}

\section{Glycemic control}

Two RCTs reported no difference between IGlar and IDet, both added to OAD, with respect to proportion of patients reaching target $\mathrm{HbA1c}$ level with either no overall $(\mathrm{RR}=1.05[0.83,1.35])$ or symptomatic hypoglycemic events $(R R=1.07 \quad[0.87,1.33])$, respectively $[65,66]$. Meta-analysis of both RCTs demonstrated comparable reduction in mean $\mathrm{HbA} 1 \mathrm{c}$ in both groups (WMD $=0.05 \%$ $[-0.07,0.16](0.5 \mathrm{mmol} / \mathrm{mol}[-0.8,1.7]))[65,66]$. The proportion of patients, who reached a target point of $\mathrm{HbA} 1 \mathrm{c} \leq 7 \% \quad(53 \mathrm{mmol} / \mathrm{mol})$, was similar between treatment arms (2 RCTs; RR $=0.95[0.86,1.06])[65,66]$.

Results of single RCTs assessing basal + bolus \pm OAD regimen revealed superiority of IGlar over IDet with respect to the primary endpoint defined as HbA1c reduction below $7 \%(53 \mathrm{mmol} / \mathrm{mol})$ with no evidence for overall hypoglycemia $(\mathrm{RR}=1.41[1.12,1.78])$, but no difference between interventions was found with respect to the number of patients achieving target $\mathrm{HbA} 1 \mathrm{c}$ without symptomatic hypoglycemia $(\mathrm{RR}=1.21[0.75,1.95])[67$, 68]. IGlar was associated with a larger reduction in mean HbA1c level (2 RCTs; WMD $=-0.25 \%$ [-0.40, -0.09$]$ $(-2.7 \mathrm{mmol} / \mathrm{mol}[-4.4 ; 1.0]))$ and allowed to reach a target $\mathrm{HbA1c}$ level $(<7 \%(53 \mathrm{mmol} / \mathrm{mol}))$ by significantly more patients when compared to IDet ( 2 RCTs; RR $=1.23$ $[1.03,1.47])[67,68]$.

\section{Hypoglycemia}

The risk of hypoglycemia in patients treated with both LAA added to OAD was comparable with respect to overall $(1 \mathrm{RCT}, \mathrm{RR}=1.05[0.93,1.19])$, symptomatic $(2$ RCTs; $\operatorname{RR}=0.99 \quad[0.90,1.08])$, severe (2 RCTs; $\mathrm{RR}=1.31 \quad[0.70,2.45])$ and nocturnal hypoglycemic events (1 RCT; RR $=0.98$ [0.77, 1.24]).

Both LAA administered according to basal + bolus \pm OAD regimen were associated with comparable risk of overall, symptomatic, severe and nocturnal hypoglycemic episodes.

\section{Weight gain}

Meta-analysis of two RCTs comparing IGlar versus IDet, both added to OAD therapy, demonstrated higher body weight gain in IGlar group $(\mathrm{WMD}=0.77 \mathrm{~kg}[0.44,1.11])$ $[65,66]$. Similarly, IGlar was also associated with a higher body weight increase as compared to IDet, when both analogs were administered together with prandial insulins (2 RCTs; WMD $=1.24 \mathrm{~kg}[0.59,1.89]$ ) [67, 68].

\section{Treatment satisfaction and quality of life}

One study comparing both LAA in basal + OAD regimen reported that IGlar was associated with a higher treatment satisfaction when compared to IDet as measured with DTSQ questionnaire (for overall result $p<0.001$ ), but no difference was shown when measured with DSC-R, WHO5 Well Being and HFS questionnaires [66].

\section{Safety}

The number of premature withdrawals due to adverse events was significantly lower in IGlar group as compared to IDet, when both interventions were administered in addition to $\mathrm{OAD}$ therapy $(\mathrm{RR}=0.40[0.24,0.69])$, but not as adjuncts to bolus insulin ( $\mathrm{RR}=0.54[0.22 ; 1,32])$. The risk of serious adverse events did not differ between both LAA administered either together with OAD (1 RCT; $\mathrm{RR}=1.26[0.87,1.83])$ or in combination with prandial insulin (2 RCTs; RR $=0.71[0.43,1.16])$. Similarly, metaanalysis of 2 RCTs demonstrated a comparable risk between IGlar and IDet in basal + bolus regimens with respect to overall adverse events $(\mathrm{RR}=1.02[0.94,1.21])$ $[67,68]$. Pooled results from two studies comparing both interventions added to OAD treatment revealed four times lower risk of application site reactions in IGlar group as compared to IDet $(\mathrm{RR}=0.22[0.07 ; 0.55])[65,66]$. Only 
one death was reported, in patient receiving IDet + OAD [65].

\section{Discussion}

Pharmacotherapy of T2DM usually starts from monotherapy with metformin, and it is further intensified by adding OADs of other classes or injectable GLP-1 agonists; nevertheless, many patients will eventually require insulin, usually beginning from one injection of basal insulin preparation [1, 2, 69]. Many insulin products have been developed so far to cover the full spectrum of T2DM patients' needs. IGlar has favorable pharmacokinetic and pharmacodynamics properties that allow providing constant insulin activity over $24 \mathrm{~h}$ with only a single injection, and is the most widely prescribed LAA [12]. Since numerous systematic reviews have been published in order to combine the outcomes of many RCTs comparing IGlar with various comparators, the additional value of our metaanalysis will be hereby discussed [16-34, 70]. Firstly, majority o earlier meta-analyses and secondary studies did not provide a full picture of clinical efficacy and safety of IGlar, since they were focused exclusively on certain aspects of insulin therapy (for example, intensive insulin treatment) [16, 17, 31]. Secondly, the other studies did not take into account the complexity of insulin treatment and pooled together trials recruiting patients with heterogenous clinical characteristics, subjected to different treatment models or accumulated results for different insulin preparations, e.g., insulin glargine with insulin detemir and premixed insulin analogs with human biphasic insulins [16, 19, 23, 25, 26, 29, 31, 33, 70]. Finally, several reviews did not attempt to accumulate included studies and presented only qualitative assessment, which significantly limited accuracy and precision of the conclusions [21, 24, 26, 28, 30, 32, 34].

In order to address the heterogeneity of T2DM and describe the efficacy and safety of investigated interventions in the context of different treatment regimens, we performed two separate analyses for each insulin preparation used for comparison. The first one involved less intensified insulin therapy (e.g., IGlar \pm OAD), while the second one concerned intensive insulin therapy (IGlar + bolus). Therefore, our analysis is comprehensive and allows us to draw very accurate and reliable conclusions. Additionally, we identified several recent primary studies, which allowed us to receive more up-to-date results and perform more thorough investigation of heterogeneity than it was previously reported.

The primary efficacy outcome was defined as the percentage of patients achieving target HbAlc level without hypoglycemia and encompassed either overall severe, nocturnal or symptomatic hypoglycemic events, as the definitions of outcomes varied between respective studies. Although the heterogeneous reporting of composite outcomes limited between-trial comparability, still the combination of glycemic control and hypoglycemia serves as the most representative measure of treatment effectiveness. Indeed, it is well known that a decrease in the HbA1c level is usually achieved at the cost of higher risk of hypoglycemic episodes. These episodes are associated with increased mortality and decreased quality of life as shown in different cohorts T2DM patients [5-7, 71]. Thus, reaching glycemic target without hypoglycemic events seems to bring particular benefits. The results of our comparative analysis indicate that IGlar is an option with favorable efficacy and acceptable safety profile. IGlar + $\mathrm{OAD}$ increased the proportion of patients reaching target HbA1c level without hypoglycemic events as compared to $\mathrm{NPH}+\mathrm{OAD}$. Although available evidence did not allow us to compare IGlar + bolus with NPH + bolus, the analysis of individual endpoints demonstrated comparable reduction of $\mathrm{HbA} 1 \mathrm{c}$ in each arm, but with concomitantly lower rate of symptomatic and nocturnal hypoglycemia in IGlar group. These results are consistent with most of the available systematic reviews comparing IGlar and NPH, which reported similar effect on HbA1c level with concomitantly lower risk of hypoglycemia for IGlar, particularly in terms of nocturnal events [18, 20, 21, 28].

Available systematic reviews showed that MIX, as compared to LAA, was associated with better glycemic control but also with a higher incidence of hypoglycemia [23, 33]. However, neither of these analyses took an effort to interpret the results in the context of relatively high degree of between-study heterogeneity with respect to treatment in the control groups. Indeed, in available RCTs patients enrolled to control groups received either monotherapy with human premixed insulins or a combination of biphasic analog insulins with OAD therapy. In the current analysis, we have shown that the superior effect of MIX + OAD over IGlar with respect to mean HbA1c decrease was associated with greater weight gain, and higher risk of symptomatic hypoglycemia. We observed an advantage of IGlar over human premixed insulins (without $\mathrm{OAD}$ ) with respect to both the reduction of $\mathrm{HbA} 1 \mathrm{c}$ and the incidence of hypoglycemia, which was not shown in previous reviews.

Finally, we also demonstrated a favorable effect of IGlar over IDet in basal + bolus regimen, as IGlar use was associated with a higher percentage of patients reaching target HbA1c without the experience of hypoglycemia. When considering basal + OAD therapy, both insulins exerted similar effect on the primary endpoint. These observations differ from those presented by other authors, which can be explained by several limitations of previous 
Table 3 Summary of the results for the comparison between IGlar and other insulin preparations

\begin{tabular}{|c|c|c|c|c|c|c|c|}
\hline \multirow[t]{2}{*}{ Outcome } & \multicolumn{2}{|l|}{ IGlar vs. NPH } & \multicolumn{3}{|l|}{ IGlar vs. MIX } & \multicolumn{2}{|l|}{ IGlar vs. IDet } \\
\hline & $+\mathrm{OAD}$ & + bolus \pm OAD & $\begin{array}{l}\text { IGlar: +OAD } \\
\text { MIX: MT }\end{array}$ & $+\mathrm{OAD}$ & $\begin{array}{l}\text { IGlar: } \\
+ \text { bolus } \pm \text { OAD } \\
\text { MIX: +OAD }\end{array}$ & $+\mathrm{OAD}$ & + bolus \pm OAD \\
\hline \multicolumn{8}{|c|}{ Target HbA1c without hypoglycemia } \\
\hline Overall & $\mathrm{n} / \mathrm{a}$ & $\mathrm{n} / \mathrm{a}$ & $\mathrm{n} / \mathrm{a}$ & No difference & $\mathrm{n} / \mathrm{a}$ & No difference & FavoursIGlar \\
\hline Nocturnal & FavoursIGlar & $\mathrm{n} / \mathrm{a}$ & FavoursIGlar & No difference & $\mathrm{n} / \mathrm{a}$ & $\mathrm{n} / \mathrm{a}$ & $\mathrm{n} / \mathrm{a}$ \\
\hline Symptomatic & $\mathrm{n} / \mathrm{a}$ & $\mathrm{n} / \mathrm{a}$ & $\mathrm{n} / \mathrm{a}$ & $\mathrm{n} / \mathrm{a}$ & $\mathrm{n} / \mathrm{a}$ & No difference & No difference \\
\hline HbA1c reduction & No difference & No difference & FavoursIGlar & Favours MIX & No difference & No difference & FavoursIGlar \\
\hline Target $\mathrm{HbA} 1 \mathrm{c}$ & No difference & No difference & FavoursIGlar & Favours MIX & FavoursIGlar & No difference & FavoursIGlar \\
\hline FPG reduction & FavoursIGlar & No difference & FavoursIGlar & No difference & No difference & FavoursIGlar & No difference \\
\hline Target FPG level & No difference & No difference & FavoursIGlar & FavoursIGlar & No difference & FavoursIGlar & No difference \\
\hline Body weight gain & No difference & No difference & No difference & FavoursIGlar & No difference & FavoursIDet & FavoursIDet \\
\hline $\begin{array}{l}\text { Treatment } \\
\text { satisfaction } \\
\text { (DTSQ) }\end{array}$ & FavoursIGlar & $\mathrm{n} / \mathrm{a}$ & No difference & $\mathrm{n} / \mathrm{a}$ & $\mathrm{n} / \mathrm{a}$ & FavoursIGlar & $\mathrm{n} / \mathrm{a}$ \\
\hline $\begin{array}{l}\text { Quality of life } \\
\text { (DSC-R) }\end{array}$ & $\mathrm{n} / \mathrm{a}$ & $\mathrm{n} / \mathrm{a}$ & $\mathrm{n} / \mathrm{a}$ & FavoursIGlar & No difference & FavoursIGlar & $\mathrm{n} / \mathrm{a}$ \\
\hline $\begin{array}{l}\text { Overall } \\
\text { hypoglycemia }\end{array}$ & No difference & No difference & FavoursIGlar & FavoursIGlar & No difference & No difference & No difference \\
\hline $\begin{array}{l}\text { Symptomatic } \\
\text { hypoglycemia }\end{array}$ & FavoursIGlar & No difference & FavoursIGlar & FavoursIGlar & No difference & No difference & No difference \\
\hline $\begin{array}{l}\text { Severe } \\
\text { hypoglycemia }\end{array}$ & No difference & No difference & No difference & No difference & No difference & No difference & No difference \\
\hline $\begin{array}{l}\text { Nocturnal } \\
\text { hypoglycemia }\end{array}$ & FavoursIGlar & FavoursIGlar & FavoursIGlar & No difference & No difference & No difference & No difference \\
\hline Overall AEs & No difference & No difference & No difference & No difference & No difference & $\mathrm{n} / \mathrm{a}$ & No difference \\
\hline $\begin{array}{l}\text { AEs associated } \\
\text { with treatment }\end{array}$ & No difference & No difference & No difference & No difference & FavoursIGlar & No difference & $\mathrm{n} / \mathrm{a}$ \\
\hline $\begin{array}{l}\text { AEs: injection site } \\
\text { reactions }\end{array}$ & No difference & No difference & $\mathrm{n} / \mathrm{a}$ & $\mathrm{n} / \mathrm{a}$ & $\mathrm{n} / \mathrm{a}$ & FavoursIGlar & $\mathrm{n} / \mathrm{a}$ \\
\hline $\begin{array}{l}\text { Withdrawals due } \\
\text { to AEs }\end{array}$ & No difference & No difference & No difference & FavoursIGlar & No difference & FavoursIGlar & No difference \\
\hline SAEs & No difference & No difference & $\mathrm{n} / \mathrm{a}$ & FavoursIGlar & No difference & No difference & No difference \\
\hline
\end{tabular}

reports that have been resolved in the current analysis. In 2008, Fakhoury et al. [17] reported an advantage of IDet over IGlar in relation to the risk of hypoglycemia, with comparable metabolic control as measured by HbAlc. However, these results were derived from an indirect comparison including evidence published before March 2007; therefore, have much less credibility than current meta-analyses performed on most up-to-date head-to-head comparisons. Another systematic review by Swinnen et al. [70] found no differences between IDet and IGlar, both in terms of glycemic control and the risk of hypoglycemia. However, the credibility of those results was limited by a high degree of statistical heterogeneity, which probably reflected between-study differences in treatment regimens. Within this analysis, we separately assessed both basa$1+\mathrm{OAD}$ and basal + bolus regimens, which was reasonable as both schemes are usually used for different disease severities. These separate analyses allowed to remove statistical heterogeneity and to obtain more precise results indicating the advantage of IGlar + bolus over IDet + bolus with respect to glycemic control.

The current review has some potential limitations. First, our meta-analysis could be criticized for choosing a fixed HbA1c target below $7.0 \%$ as a component of the composite primary endpoint, while the individualization is an important element of the contemporary diabetes management. Nevertheless, most major clinical guidelines maintained HbA1c level of $<7.0 \%$ as a general treatment goal, which can be advantageous for majority of patients with diabetes [72-74]. The treatment target may be relieved mainly in patients with long-lasting diabetes, short life expectancy, existing comorbidities, etc. Since most studies included in our meta-analysis excluded individuals for whom less stringent glycemic control target could be 
applied, such as elderly individuals with severe chronic complications, the definition of the primary endpoint adopted in this paper is justified. Additionally, due to the short duration of studies included in the current metaanalysis and low incidence of malignancies, we were not able to analyze between-treatment differences in the risk of malignancies. Such analysis would have been of potential clinical importance taking into account earlier studies searching for potential association between T2DM and pharmacological therapies used for its treatment and the risk of cancer [75-81].

As depicted on a study flow diagram, 363 papers did not meet inclusion criteria for the analysis due to various reasons. This may raise concerns whether the data selection process was correct. Such a high number of publications excluded from the meta-analysis are to some extent a consequence of a highly sensitive strategy used for database searching, which allowed us to retrieve all important studies. This also, however, resulted in a relatively large number of less relevant data including reviews, letters, conference proceedings and others, which had to be removed in further steps of selection process.

Finally, the quality of included studies, which were mainly designed as open-labeled comparisons, should be discussed. Of note, proper maintenance of glycemic control requires continuous insulin titration, which could not be performed when patients or physicians are unaware of the assigned treatment since various insulins require different dosing. Blinding to treatment allocation would lead to suboptimal glucose levels control with excessive rate of hypoglycemia, and therefore, open-labeled design is justified in studies assessing insulin therapy.

Finally, yet another limitation of this review is a short follow-up in the majority of included RCTs. Most studies had a duration up to 6 months, which may not provide a fully reliable picture of relative efficacy of insulin treatment in long-term perspective. The only longitudinal RCT identified within our systematic review was designed to compare IGlar versus NPH, both added to OAD therapy [51]. Nevertheless, due to the long follow-up, the protocol allowed for modification of both OAD therapy and insulin treatment, so that prandial insulins could be introduced or withdrawn during the study. Indeed, during the mean follow-up of 260 weeks, most of the patients in both treatment groups received human prandial insulins. The study demonstrated a difference in $\mathrm{HbA} 1 \mathrm{c}$ reduction in favor of twice daily NPH insulin, which was most likely due to higher insulin dose and higher percentage of patients who were co-administered with prandial insulin in NPH group. Indeed, a post hoc analysis for patients treated solely with basal insulins and OAD demonstrated nearly equivalent reduction of $\mathrm{HbA1c}$ in both groups [51]. Additionally, IGlar was associated with fewer patients experiencing severe hypoglycemia and with no apparent difference in mean body gain [51].

In conclusion, for the majority of examined efficacy and safety outcomes, IGlar use in T2DM patients was superior or at least non-inferior to the alternative insulin treatment options (Table 3).

Acknowledgments We would like to thank Katarzyna Kapcia for assistance with graphic design and editorial support and Kinga Rup for linguistic help in the preparation of this manuscript. This study was supported by a scientific grant from Sanofi Poland to HTA Consulting.

Conflict of interest P.R, P.W, A.R-S. and G.N. are employees of HTA Consulting, the company that received grant from Sanofi Poland for this project. HTA Consulting received also grants from Novo Nordisk Pharma Poland for other scientific projects. J.L and A.S. are employees of Sanofi Poland. MTM received remuneration for lectures and Advisory Board membership.

Human and Animal Rights disclosure This article does not contain any studies with human or animal subjects performed by the any of the authors.

Open Access This article is distributed under the terms of the Creative Commons Attribution License which permits any use, distribution, and reproduction in any medium, provided the original author(s) and the source are credited.

\section{References}

1. Inzucchi SE, Bergenstal RM, Buse JB et al (2012) Management of hyperglycaemia in type 2 diabetes: a patient-centered approach. Position statement of the American Diabetes Association (ADA) and the European Association for the Study of Diabetes (EASD). Diabetologia 55:1577-1596

2. Handelsman Y, Mechanick JI, Blonde L et al (2011) American Association of Clinical Endocrinologists Medical Guidelines for Clinical Practice for developing a diabetes mellitus comprehensive care plan. Endocr Pract 17:1-53

3. U.K. Prospective Diabetes Study Group (1995) U.K. prospective diabetes study 16. Overview of 6 years' therapy of type II diabetes: a progressive disease. Diabetes 44:1249-1258

4. Control Group, Turnbull FM, Abraira C et al (2009) Intensive glucose control and macrovascular outcomes in type 2 diabetes. Diabetologia 52:2288-2298. doi:10.1007/s00125-009-1470-0

5. Zoungas S, Patel A, Chalmers J et al (2010) Severe hypoglycemia and risks of vascular events and death. $\mathrm{N}$ Engl $\mathrm{J}$ Med 363:1410-1418. doi:10.1056/NEJMoa1003795

6. Hsu P-F, Sung S-H, Cheng H-M et al (2013) Association of clinical symptomatic hypoglycemia with cardiovascular events and total mortality in type 2 diabetes: a nationwide populationbased study. Diabetes Care 36:894-900. doi:10.2337/dc12-0916

7. Gitt AK, Bramlage P, Binz C et al (2012) Hypoglycaemia is more frequent in type 2 diabetic patients with co-morbid vascular disease: an analysis of the DiaRegis registry. Eur J Prev Cardiol 19:765-772. doi:10.1177/1741826711411104

8. Nathan DM, Buse JB, Davidson MB et al (2009) Medical management of hyperglycemia in type 2 diabetes: a consensus algorithm for the initiation and adjustment of therapy: a consensus statement of the American Diabetes Association and the European Association for the Study of Diabetes. Diabetes Care 32:193-203. doi:10.2337/dc08-9025 
9. National Collaborating Centre for Chronic Conditions (2008) Type 2 diabetes: national clinical guideline for management in primary and secondary care (update). Royal College of Physicians, London. http://www.nice.org.uk/guidance/cg66

10. Scottish Intercollegiate Guidelines Network (2010) Management of diabetes. A national clinical guideline. Scottish Intercollegiate Guidelines Network (SIGN), Edinburgh, Scotland. http://www. sign.ac.uk/pdf/sign116.pdf

11. IDF, ISPAD (2011) Global IDF/ISPAD guideline for diabetes in childhood and adolescence

12. Lepore M, Pampanelli S, Fanelli C et al (2000) Pharmacokinetics and pharmacodynamics of subcutaneous injection of long-acting human insulin analog glargine, NPH insulin, and ultralente human insulin and continuous subcutaneous infusion of insulin lispro. Diabetes 49:2142-2148

13. Gerich J, Becker RHA, Zhu R, Bolli GB (2006) Fluctuation of serum basal insulin levels following single and multiple dosing of insulin glargine. Diabetes Technol Ther 8:237-243. doi:10.1089/ dia.2006.8.237

14. Rotenstein LS, Ran N, Shivers JP et al (2012) Opportunities and challenges for biosimilars: What's on the horizon in the global insulin market? Clin Diabetes 30:138-150. doi:10.2337/diaclin. 30.4 .138

15. Pistrosch F, Köhler C, Schaper F et al (2013) Effects of insulin glargine versus metformin on glycemic variability, microvascular and beta-cell function in early type 2 diabetes. Acta Diabetol 50:587-595. doi:10.1007/s00592-012-0451-9

16. Esposito K, Chiodini P, Capuano A et al (2012) Basal supplementation of insulin lispro protamine suspension versus insulin glargine and detemir for type 2 diabetes: meta-analysis of randomized controlled trials. Diabetes Care 35:2698-2705. doi:10. 2337/dc12-0698

17. Fakhoury W, Lockhart I, Kotchie RW et al (2008) Indirect comparison of once daily insulin detemir and glargine in reducing weight gain and hypoglycaemic episodes when administered in addition to conventional oral anti-diabetic therapy in patients with type-2 diabetes. Pharmacology 82:156-163. doi:10.1159/000149569

18. Mullins P, Sharplin P, Yki-Jarvinen H et al (2007) Negative binomial meta-regression analysis of combined glycosylated hemoglobin and hypoglycemia outcomes across eleven Phase III and IV studies of insulin glargine compared with neutral protamine Hagedorn insulin in type 1 and type 2 diabetes mellitus. Clin Ther 29:1607-1619. doi:10.1016/j.clinthera.2007.08.020

19. Rosenstock J, Dailey G, Massi-Benedetti M et al (2005) Reduced hypoglycemia risk with insulin glargine: a meta-analysis comparing insulin glargine with human NPH insulin in type 2 diabetes. Diabetes Care 28:950-955

20. Bazzano LA, Lee LJ, Shi L et al (2008) Safety and efficacy of glargine compared with NPH insulin for the treatment of Type 2 diabetes: a meta-analysis of randomized controlled trials. Diabet Med 25:924-932. doi:10.1111/j.1464-5491.2008.02517.x

21. Clissold R, Clissold S (2007) Insulin glargine in the management of diabetes mellitus: an evidence-based assessment of its clinical efficacy and economic value. Core Evid 2:89-110

22. Dailey G, Admane K, Mercier F, Owens D (2010) Relationship of insulin dose, A1c lowering, and weight in type 2 diabetes: comparing insulin glargine and insulin detemir. Diabetes Technol Ther 12:1019-1027. doi:10.1089/dia.2010.0063

23. Fonseca V, Davidson J, Home P et al (2010) Starting insulin therapy with basal insulin analog or premix insulin analog in T2DM: a pooled analysis of treat-to-target trials. Curr Med Res Opin 26:1621-1628. doi:10.1185/03007995.2010.485087

24. Halimi S, Raskin P, Liebl A et al (2005) Efficacy of biphasic insulin aspart in patients with type 2 diabetes. Clin Ther 27:S57-S74

25. Horvath K, Jeitler K, Berghold A, Ebrahim SH, Gratzer TW, Plank J, Kaiser T, Pieber TR, Siebenhofer A (2007) Long-acting insulin analogues versus NPH insulin (human isophane insulin) for type 2 diabetes mellitus. Cochrane Database Syst Rev 2:CD005613. doi:10.1002/14651858.CD005613.pub3

26. Ilag LL, Kerr L, Malone JK, Tan MH (2007) Prandial premixed insulin analogue regimens versus basal insulin analogue regimens in the management of type 2 diabetes: an evidence-based comparison. Clin Ther 29:1254-1270

27. Jansen HJ, Vervoort G, van der Graaf M, Tack CJ (2010) Pronounced weight gain in insulin-treated patients with type 2 diabetes mellitus is associated with an unfavourable cardiometabolic risk profile. Neth J Med 68:359-366

28. Levien TL, Baker DE, White JR Jr, Campbell RK (2002) Insulin glargine: a new basal insulin. Ann Pharmacother 36:1019-1027

29. Monami M, Marchionni N, Mannucci E (2008) Long-acting insulin analogues versus NPH human insulin in type 2 diabetes: a meta-analysis. Diabetes Res Clin Pract 81:184-189. doi:10.1016/ j.diabres.2008.04.007

30. Pollock RF, Erny-Albrecht KM, Kalsekar A, Bruhn D, Valentine WJ (2011) Long-acting insulin analogs: a review of "RealWorld" effectiveness in patients with type 2 diabetes. Curr Diabetes Rev 7(1):61-74. doi:10.2174/157339911794273892

31. Pontiroli AE, Miele L, Morabito A (2012) Metabolic control and risk of hypoglycaemia during the first year of intensive insulin treatment in type 2 diabetes: systematic review and meta-analysis. Diabetes Obes Metab 14:433-446

32. Rolla AR, Rakel RE (2005) Practical approaches to insulin therapy for type 2 diabetes mellitus with premixed insulin analogues. Clin Ther 27:1113-1125. doi:10.1016/j.clinthera.2005.07.003

33. Qayyum R, Bolen S, Maruthur N et al (2008) Systematic review: comparative effectiveness and safety of premixed insulin analogues in type 2 diabetes. Ann Intern Med 149:549-559

34. Gough SCL (2007) A review of human and analogue insulin trials. Diabetes Res Clin Pract 77:1-15. doi:10.1016/j.diabres. 2006.10.015

35. Moher D, Liberati A, Tetzlaff J et al (2009) Preferred reporting items for systematic reviews and meta-analyses: the PRISMA statement. PLoS Med 6:e1000097. doi:10.1371/journal.pmed. 1000097

36. Davidson J, Lacaya L, Jiang $H$ et al (2010) Impact of race/ethnicity on the efficacy and safety of commonly used insulin regimens: a post hoc analysis of clinical trials in type 2 diabetes mellitus. Endocr Pract 16:818-828. doi:10.4158/EP09285.OR

37. Jadad AR, Moore RA, Carroll D et al (1996) Assessing the quality of reports of randomized clinical trials: is blinding necessary? Control Clin Trials 17:1-12

38. Higgins JPT, Green S (eds) (2011) Cochrane handbook for systematic reviews of interventions version 5.1.0 [updated March 2011]. The Cochrane Collaboration. http://www.cochrane-hand book.org

39. Hsia SH (2011) Insulin glargine compared to NPH among insulin-naïve, U.S. inner city, ethnic minority type 2 diabetic patients. Diabetes Res Clin Pract 91:293-299. doi:10.1016/j.diabres.2010. 11.028

40. Forst T, Larbig M, Hohberg C et al (2010) Adding insulin glargine vs. NPH insulin to metformin results in a more efficient postprandial beta-cell protection in individuals with type 2 diabetes. Diabetes Obes Metab 12:437-441. doi:10.1111/j.14631326.2010.01209.x

41. Eliaschewitz FG, Calvo C, Valbuena H et al (2006) Therapy in type 2 diabetes: insulin glargine vs. NPH insulin both in combination with glimepiride. Arch Med Res 37:495-501

42. Fritsche A, Schweitzer MA, Häring H-U (2003) Glimepiride combined with morning insulin glargine, bedtime neutral protamine hagedorn insulin, or bedtime insulin glargine in patients with type 2 diabetes. A randomized, controlled trial. Ann Intern Med 138:952-959 
43. Yki-Järvinen $H$, Kauppinen-Mäkelin $\mathrm{R}$, Tiikkainen $\mathrm{M}$ et al (2006) Insulin glargine or NPH combined with metformin in type 2 diabetes: the LANMET study. Diabetologia 49:442-451. doi:10.1007/s00125-005-0132-0

44. Riddle MC, Rosenstock J, Gerich J (2003) The treat-to-target trial: randomized addition of glargine or human NPH insulin to oral therapy of type 2 diabetic patients. Diabetes Care 26:3080-3086

45. Massi Benedetti M, Humburg E, Dressler A, Ziemen M (2003) A one-year, randomised, multicentre trial comparing insulin glargine with NPH insulin in combination with oral agents in patients with type 2 diabetes. Horm Metab Res 35:189-196. doi:10.1055/ s-2003-39080

46. Koivisto V, Cleall S, Pontiroli AE, Giugliano D (2011) Comparison of insulin lispro protamine suspension versus insulin glargine once daily in basal-bolus therapies with insulin lispro in type 2 diabetes patients: a prospective randomized open-label trial. Diabetes Obes Metab 13:1149-1157

47. Rosenstock J, Schwartz SL, Clark CM Jr et al (2001) Basal insulin therapy in type 2 diabetes: 28-week comparison of insulin glargine (HOE 901) and NPH insulin. Diabetes Care 24:631-636

48. Esposito K, Ciotola M, Maiorino MI et al (2008) Addition of neutral protamine lispro insulin or insulin glargine to oral type 2 diabetes regimens for patients with suboptimal glycemic control: a randomized trial. Ann Intern Med 149:531-539

49. Strojek K, Shi C, Carey MA, Jacober SJ (2010) Addition of insulin lispro protamine suspension or insulin glargine to oral type 2 diabetes regimens: a randomized trial. Diabetes Obes Metab 12:916-922

50. Roach P, Woodworth JR (2002) Clinical pharmacokinetics and pharmacodynamics of insulin lispro mixtures. Clin Pharmacokinet 41:1043-1057

51. Rosenstock J, Fonseca V, McGill JB et al (2009) Similar progression of diabetic retinopathy with insulin glargine and neutral protamine Hagedorn (NPH) insulin in patients with type 2 diabetes: a long-term, randomised, open-label study. Diabetologia 52:1778-1788. doi:10.1007/s00125-009-1415-7

52. Al-Shaikh AR (2006) Comparison of basal insulin added to oral agents versus twice-daily premixed insulin as initial insulin therapy for type 2 diabetes. Pak J Med Sci 22:14-17

53. Janka HU, Plewe G, Riddle MC et al (2005) Comparison of basal insulin added to oral agents versus twice-daily premixed insulin as initial insulin therapy for type 2 diabetes. Diabetes Care 28:254-259

54. Schiel R, Muller UA (2007) Efficacy and treatment satisfaction of once-daily insulin glargine plus one or two oral antidiabetic agents versus continuing premixed human insulin in patients with type 2 diabetes previously on long-term conventional insulin therapy: the Switch pilot study. Exp Clin Endocrinol Diabetes 115:627-633

55. Buse JB, Wolffenbuttel BH, Herman WH et al (2009) DURAbility of basal versus lispro mix 75/25 insulin efficacy (DURABLE) trial 24-week results: safety and efficacy of insulin lispro mix $75 / 25$ versus insulin glargine added to oral antihyperglycemic drugs in patients with type 2 diabetes. Diabetes Care 32:1007-1013

56. Kann PH, Wascher T, Zackova V et al (2006) Starting insulin therapy in type 2 diabetes: twice-daily biphasic insulin Aspart 30 plus metformin versus once-daily insulin glargine plus glimepiride. Exp Clin Endocrinol Diabetes 114:527-532. doi:10.1055/s2006-949655

57. Raskin P, Allen E, Hollander $P$ et al (2005) Initiating insulin therapy in type 2 Diabetes: a comparison of biphasic and basal insulin analogs. Diabetes Care 28:260-265

58. Robbins DC, Beisswenger PJ, Ceriello A et al (2007) Mealtime $50 / 50$ basal + prandial insulin analogue mixture with a basal insulin analogue, both plus metformin, in the achievement of target $\mathrm{HbA} 1 \mathrm{c}$ and pre- and postprandial blood glucose levels in patients with type 2 diabetes: a multinational, 24-week, randomized, open-label, parallel-group comparison. Clin Ther 29:2349-2364

59. Strojek K, Bebakar W, Khutsoane D et al (2009) Once-daily initiation with biphasic insulin aspart 30 versus insulin glargine in patients with type 2 diabetes inadequately controlled with oral drugs: an open-label, multinational RCT. Curr Med Res Opin 28:2887-2894

60. Bowering K, Reed VA, Felicio J et al (2012) A study comparing insulin lispro mix 25 with glargine plus lispro therapy in patients with Type 2 diabetes who have inadequate glycaemic control on oral anti-hyperglycaemic medication: results of the PARADIGM study. Diabet Med 29:e263-e272

61. Miser WF, Arakaki R, Jiang H et al (2010) Randomized, openlabel, parallel-group evaluations of basal-bolus therapy versus insulin lispro premixed therapy in patients with type 2 diabetes mellitus failing to achieve control with starter insulin treatment and continuing oral antihyperglycemic drugs: a noninferiority intensification substudy of the DURABLE trial. Clin Ther 32:896-908

62. Fritsche A, Larbig M, Owens D, Haring HU (2010) Comparison between a basal-bolus and a premixed insulin regimen in individuals with type 2 diabetes-results of the GINGER study. Diabetes Obes Metab 12:115-123

63. Jain SM, Mao X, Escalante-Pulido M et al (2010) Prandial-basal insulin regimens plus oral antihyperglycaemic agents to improve mealtime glycaemia: initiate and progressively advance insulin therapy in type 2 diabetes. Diabetes Obes Metab 12:967-975

64. Rosenstock J, Ahmann AJ, Colon G et al (2008) Advancing insulin therapy in type 2 diabetes previously treated with glargine plus oral agents: prandial premixed (insulin lispro protamine suspension/lispro) versus basal/bolus (glargine/lispro) therapy. Diabetes Care 31:20-25

65. Rosenstock J, Davies M, Home PD et al (2008) A randomised, 52-week, treat-to-target trial comparing insulin detemir with insulin glargine when administered as add-on to glucose-lowering drugs in insulin-naive people with type 2 diabetes. Diabetologia 51:408-416. doi:10.1007/s00125-007-0911-x

66. Swinnen SG, Dain M-P, Aronson R et al (2010) A 24-week, randomized, treat-to-target trial comparing initiation of insulin glargine once-daily with insulin detemir twice-daily in patients with type 2 diabetes inadequately controlled on oral glucoselowering drugs. Diabetes Care 33:1176-1178. doi:10.2337/dc092294

67. Hollander P, Cooper J, Bregnhøj J, Pedersen CB (2008) A 52-week, multinational, open-label, parallel-group, noninferiority, treat-to-target trial comparing insulin detemir with insulin glargine in a basal-bolus regimen with mealtime insulin aspart in patients with type 2 diabetes. Clin Ther 30:1976-1987. doi:10. 1016/j.clinthera.2008.11.001

68. Raskin P, Gylvin T, Weng W, Chaykin L (2009) Comparison of insulin detemir and insulin glargine using a basal-bolus regimen in a randomized, controlled clinical study in patients with type 2 diabetes. Diabetes Metab Res Rev 25:542-548. doi:10.1002/ dmrr.989

69. Saisho Y, Kou K, Tanaka K et al (2013) Postprandial serum $\mathrm{C}$-peptide to plasma glucose ratio predicts future insulin therapy in Japanese patients with type 2 diabetes. Acta Diabetol 50:987-988. doi:10.1007/s00592-012-0441-y

70. Swinnen SG, Simon AC, Holleman F et al (2011) Insulin detemir versus insulin glargine for type 2 diabetes mellitus. Cochrane Database Syst Rev. doi:10.1002/14651858.CD006383.pub2

71. Piatti PM, Marone E, Mantero M et al (2013) Effect of normalization of fasting glucose by intensified insulin therapy and influence of eNOS polymorphisms on the incidence of restenosis after peripheral angioplasty in patients with type 2 diabetes: 
a randomized, open-label clinical trial. Acta Diabetol 50:373-382. doi:10.1007/s00592-012-0426-x

72. American Diabetes Association (2013) Standards of medical care in diabetes-2014. Diabetes Care 37:S14-S80. doi:10.2337/dc14-S014

73. Authors/Task Force Members, Rydén L, Grant PJ et al (2013) ESC Guidelines on diabetes, pre-diabetes, and cardiovascular diseases developed in collaboration with the EASD: the Task Force on diabetes, pre-diabetes, and cardiovascular diseases of the European Society of Cardiology (ESC) and developed in collaboration with the European Association for the Study of Diabetes (EASD). Eur Heart J 34:3035-3087. doi:10.1093/eur heartj/eht108

74. International Diabetes Federation (2012) Clinical Guidelines Task Force. Global guideline for type 2 diabetes. http://www.idf. org/global-guideline-type-2-diabetes-2012

75. Nicolucci A (2010) Epidemiological aspects of neoplasms in diabetes. Acta Diabetol 47:87-95. doi:10.1007/s00592-010-0187-3

76. Janghorbani M, Dehghani M, Salehi-Marzijarani M (2012) Systematic review and meta-analysis of insulin therapy and risk of cancer. Horm Cancer 3:137-146. doi:10.1007/s12672-012-0112-z
77. Colmers IN, Bowker SL, Tjosvold LA, Johnson JA (2012) Insulin use and cancer risk in patients with type 2 diabetes: a systematic review and meta-analysis of observational studies. Diabetes Metab 38:485-505. doi:10.1016/j.diabet.2012.08.011

78. Chen Y, Chen Q, Wang Z, Zhou J (2013) Insulin therapy and risk of prostate cancer: a systematic review and meta-analysis of observational studies. PLoS ONE 8:e81594. doi:10.1371/journal. pone.0081594

79. Karlstad O, Starup-Linde J, Vestergaard P et al (2013) Use of insulin and insulin analogs and risk of cancer-systematic review and meta-analysis of observational studies. Curr Drug Saf 8:333-348

80. Tseng C-H (2013) Diabetes, insulin use, smoking, and pancreatic cancer mortality in Taiwan. Acta Diabetol 50:879-886. doi:10. 1007/s00592-013-0471-0

81. Gerstein HC, Bosch J, Dagenais GR et al (2012) Basal insulin and cardiovascular and other outcomes in dysglycemia. N Engl J Med 367:319-328. doi:10.1056/NEJMoa1203858 